\title{
Maximization of energy absorption for a wave energy converter using the
}

\section{deep machine learning}

\author{
Liang Li, Zhiming Yuan", Yan Gao \\ Department of Naval Architecture, Ocean and Marine Engineering, University of Strathclyde, 100 Montrose Street, \\ Glasgow, G4 OLZ, UK \\ *Corresponding author: zhiming.yuan@strath.ac.uk.
}

\begin{abstract}
A controller is usually used to maximize the energy absorption of wave energy converter. Despite the development of various control strategies, the practical implementation of wave energy control is still difficult since the control inputs are the future wave forces. In this work, the artificial intelligence technique is adopted to tackle this problem. A multi-layer artificial neural network is developed and trained by the deep machine learning algorithm to forecast the short-term wave forces. The model predictive control strategy is used to implement real-time latching control action to a heaving pointabsorber. Simulation results show that the average energy absorption is increased substantially with the controller. Since the future wave forces are predicted, the controller is applicable to a full-scale wave energy converter in practice. Further analysis indicates that the prediction error has a negative effect on the control performance, leading to the reduction of energy absorption.
\end{abstract}

Keywords: wave energy converter; wave energy control; energy absorption; neural network; deep machine learning; wave force prediction.

\section{Introduction}

To keep up with the growth of global energy demand, various energy systems have been developed to extract power from marine energy sources (offshore wind, ocean waves, tide, etc) [1-3]. Compared with other marine energy resources, wave energy is a kind of resource with high power density and all-day availability. Owing to these advantages, wave energy is regarded as a prospective solution to the sustainable generation of power. The device used to harvest energy from ocean waves is called the wave energy converter (WEC). Li et al. [4] showed the power output of an oscillatingbody WEC installed on a spar-type floating wind turbine. He et al. [5] utilized a floater breakwater to harvest energy from the waves. Experimental study of the concept was performed. Falcao and Henriques [6] presented a review on the oscillating-water-column WEC. Stansby et al. [7] examined the dynamics of multi-float WEC concept M4.

Although a set of WEC concepts have been developed, the energy harvesting efficiency is still not satisfactory, especially in the off-resonance state. One of the solutions is the usage of a non-linear power take-off (PTO) system. Zhang and Yang [8] showed that a PTO system with nonlinear spring could harvest more energy in random waves. Xiao et al. [9] investigated the power capture of an 
oscillating-body WEC with three different PTO systems. They showed that the nonlinear behavior of the PTO was beneficial to the power capture. An more widely accepted approach is to regulate the WEC dynamics with a controller. Babarit et al. [10] studied how the declutching control influenced the energy absorption of a WEC in regular and irregular waves. Tom et al. [11] optimized the power capture of an oscillating surge WEC using the pseudo-spectral control method. The latching control was firstly introduced by Budal and Falnes [12]. They found that one condition for maximizing energy absorption was to keep the velocity in phase with the wave excitation force. Inspired by their pioneering work, many researchers begin to adopt the latching control to enhance wave energy efficiency. Babarit and Clement [13] assessed the benefits produced by the latching control. Based on the pre-generated wave elevations, the optimal command theory was applied to derive the control command. Henriques et al. [14] applied the latching control to an oscillating-water-column WEC. Until now, the WEC control studies mainly concentrate on the development of control strategy whereas the practical application of control is seldom reported. It is mainly because the implementation of the controll to a realistic WEC requires the prediction of future wave forces.

Given the explosive growth of the artificial intelligence, the deep machine learning algorithm based on the artificial neural network has been widely used for regression and classification. The artificial neural network was firstly proposed by Mcculloch and Pitts [15]. At that time, the structure of the neural network was very simple since the inference between densely connected nets with many hidden layers is rather difficult. In 2006, Hinton et al. [16] proposed a fast, greedy algorithm for the multi-layers network. Their work marked the era of 'deep learning'. Although the deep machine learning is basically employed in the recognition and interaction of signal, it is also powerful for prediction in many fields. Lv et al. [17] used the deep learning approach to predict the traffic flow. Islam and Morimoto [18] forecasted the inside air temperature of a pillar cooler with the neural network. Recently, the machine learning was introduced to marine hydrodynamic prediction. Pourzangbar et al. [19] predicted scour of breakwaters using the genetic programming and the artificial neural network, respectively. Ebtehaj et al. [20] developed an integrated framework of learning machines to predict scour at pile groups.

The present study is aimed at developing a real-time controller applicable in practice by considering the short-term wave force forecasting. An artificial neural network is developed for the wave force prediction. The neural network is trained with the deep machine learning algorithm to learn the underlying relationship between wave forces in the past and future wave forces. The smart controller is implemented to a heaving point-absorber to maximize the energy absorption. The advantage of the neural network against traditional prediction method will be discussed.

\section{Numerical model}

Fig. 1 gives the sketch of the heaving point-absorber WEC studied in this work. The pointabsorber consists of a floater harvesting wave energy and a PTO system conversing wave energy into 
electrical power. The floater, a hemisphere with a radius of $5 \mathrm{~m}$, is rigidly connected to the PTO system fixed at the seabed. Only heave motion of the floater is allowed.

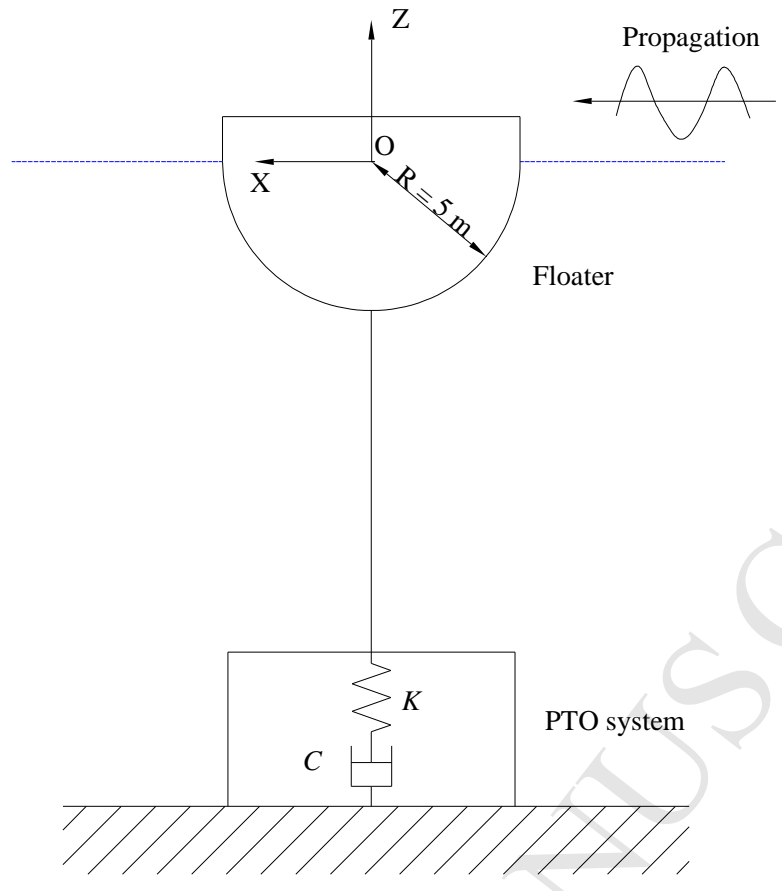

Fig. 1. Wave energy converter.

The generator force is modeled with a linear damping coefficient $C$ and stiffness $K$. According to Vicente et al. [21], the stiffness of a PTO system is typically around ten percent of the hydrostatic coefficient. Therefore, $K=0.1 \rho \mathrm{g} \pi R^{2}$ is adopted. $\rho$ is the water density and $\mathrm{g}$ is the acceleration of gravity. Fig. 2 illustrates the sensitivity of the PTO system to wave frequency $\omega$ and damping coefficient $C$. To harvest as much energy as possible, $C=8.14 \times 10^{5} \mathrm{~kg} / \mathrm{s}$ is used. The controller regulates the WEC response by locking and releasing the floater alternately following a certain rule. A very large but finite damping coefficient $c$ is used to lock the floater. This kind of control is widely known as the latching control.

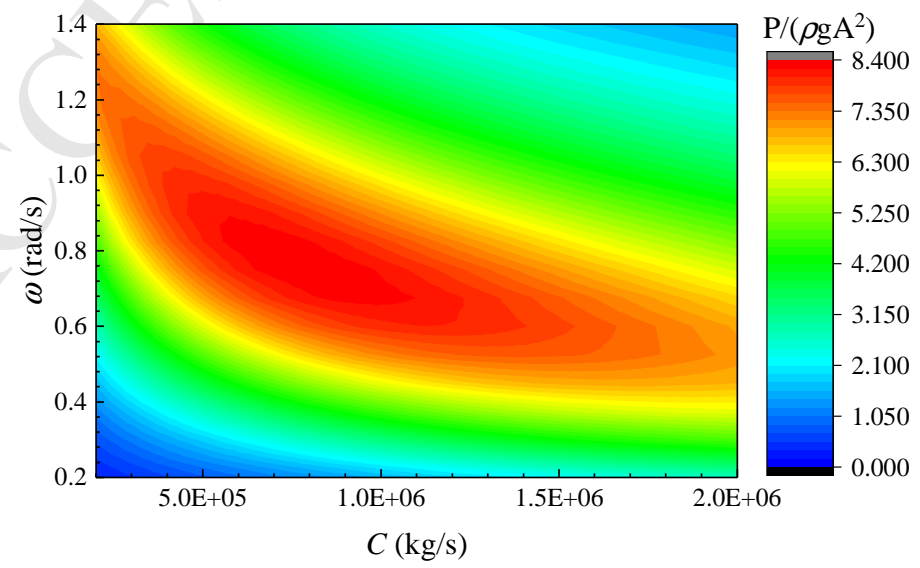

Fig. 2. The sensitivity of energy absorption to wave frequency and damping coefficient $C$ in regular waves. Wave amplitude $A=1 \mathrm{~m}$. 
The linear potential flow theory is adopted to address the wave-structure interaction. The viscous effect is not considered. It is worth noting that the linear dynamic model is invalid in extreme sea state, where the free surface condition involves strong nonlinearity. Since a full-scale WEC just works in moderate sea state, the linear dynamic model is still applicable. A right-handed coordinate system fixed to the earth is used (see Fig. 1). The center of the coordinate system is fixed at the mean sea surface. $Z$ axial is positive upward. $X$ axial is along the propagation direction of the sea waves.

Based on the impulse response theory [22], the time-domain motion equation of the floater is given by

$$
(M+m) \ddot{z}(t)+\int_{0}^{t} H(t-\tau) \dot{z}(\tau) d \tau+\rho g \pi R^{2} z(t)=F_{\text {wave }}(t)-C \dot{z}(t)-K z(t)-\beta(t) c \dot{z}(t)
$$

where $M$ is the mass of the floater and $m$ is the added mass corresponding to infinite frequency. $z, \dot{z}$ and $\ddot{z}$ are the displacement, velocity and acceleration. $F_{\text {wave }}$ is the wave excitation force. $\beta(t)$ is the binary control sequence. When $\beta=1$, the floater is locked; when $\beta=0$, it is free to oscillate. The point-absorber switches abruptly between two states $(\beta=0,1)$ so that the latching control is a bangbang control. $H$ is the so-called retardation kernel function which represents the memory effect of radiation force. It can be obtained either from the added mass $a(\omega)$ or the potential damping $b(\omega)$

$$
H(t)=\frac{2}{\pi} \int_{0}^{\infty} \frac{b(\omega)}{\omega} \sin (\omega t) d \omega=\frac{2}{\pi} \int_{0}^{\infty} a(\omega) \cos (\omega t) d \omega
$$

Although Eq. (1) is widely used to simulate the wave-structure interaction, such form makes it inconvenient to implement the control strategy. An alternative model is thus developed to simulate the dynamics of the point-absorber in random waves, in which the convolution term is replaced by a statespace representation.

$$
\begin{aligned}
& \int_{0}^{t} H(t-\tau) \dot{z}(\tau) d \tau=\overrightarrow{\boldsymbol{C}} \boldsymbol{u}(t) \\
& \dot{\boldsymbol{u}}(t)=\overrightarrow{\boldsymbol{A}} \boldsymbol{u}(t)+\overrightarrow{\boldsymbol{B}} \dot{z}(t)
\end{aligned}
$$

where $\overrightarrow{\boldsymbol{A}}, \overrightarrow{\boldsymbol{B}}$ and $\overrightarrow{\boldsymbol{C}}$ are constant matrices identifying the system with dimensions $n \times n, n \times 1$ and $1 \times n . n$ is the order of the system. These matrices can be derived from the hydrodynamic coefficients of the point-absorber by system identification. $\boldsymbol{u}$ is an intermediate vector with dimension $n \times 1$. Define a state vector $\boldsymbol{x}=\left[z, \dot{z}, \boldsymbol{u}^{T}\right]^{T}$ with dimension $(n+2) \times 1$. Then Eq. (1) can be re-expressed as

$$
\begin{aligned}
& \dot{\boldsymbol{x}}=\boldsymbol{\gamma} \cdot \boldsymbol{x}+\boldsymbol{\eta} \\
& \boldsymbol{\gamma}=\left[\begin{array}{ccc}
0 & 1 & \mathbf{0} \\
-\frac{\rho g \pi R^{2}+K}{M+m} & -\frac{C+\beta c}{M+m} & -\frac{\overrightarrow{\boldsymbol{C}}}{M+m} \\
\mathbf{0} & \overrightarrow{\boldsymbol{B}} & \overrightarrow{\boldsymbol{A}}
\end{array}\right], \boldsymbol{\eta}=\left[\begin{array}{c}
0 \\
\frac{F_{\text {wave }}}{M+m} \\
\mathbf{0}
\end{array}\right]
\end{aligned}
$$

Eq. (4) is a first-order, one-variable differential formula, which is easier to handle. Given the initial condition $\boldsymbol{x}(0)=\mathbf{0}$, it becomes a classical initial-value problem and the time series of floater 
114 movement can be obtained by the $4^{\text {th }}$ Runge-Kutta method. Then, the average energy absorption

115 during simulation interval $[0, T]$ is given by

$$
P=\frac{1}{T} \int_{0}^{T} C \cdot \dot{z}(t, \beta)^{2} d t
$$

The random sea waves can be efficiently approximated by a set of regular wave components with

118 various frequencies and random phases

$$
\begin{aligned}
& \xi(t)=\operatorname{Re}\left[\sum_{j=1}^{N} A_{j} e^{i\left(\omega_{j} t+\varepsilon_{j}\right)}\right] \\
& A_{j}=\sqrt{2 S\left(\omega_{j}\right) \square \omega}
\end{aligned}
$$

where $A_{j}, \omega_{j}$, and $\varepsilon_{j}$ are the amplitude, frequency and random phase of the regular wave component $j$. $S(\omega)$ is the wave spectrum adopted to describe the statistical feature of the random waves. $N$ is the number of regular wave components in the wave spectrum. If $\omega_{j}$ is uniformly distributed over the wave frequency range, the stochastic wave elevations will start to repeat after a certain duration [23]. To address this issue, the correction technique in Ref [24] is adopted here. The wave frequency range is first uniformly divided into $N$ segments and $\omega_{j}$ is afterward randomly distributed within segment $j$. Given the time series of random wave elevations, the linear wave forces are obtained with the firstorder transfer function $\Psi$.

$$
F_{\text {wave }}(t)=\operatorname{Re}\left[\sum_{j=1}^{N} \psi\left(\omega_{j}\right) A_{j} e^{i\left(\omega_{j} t+\varepsilon_{j}\right)}\right]
$$

\section{Control strategy}

\subsection{Optimal command theory}

The objective of latching control is to maximize the average energy absorption through the binary control sequence $\beta(t)$

$$
\max P=\frac{1}{T} \int_{0}^{T} C \cdot \dot{z}(t, \beta)^{2} d t
$$

From a mathematical point of view, it is required to find the maximum of $P$ subject to constraint Eq. (4). If the incident wave is regular, it becomes an impedance matching problem and can be solved analytically [13]. Otherwise, the solution is non-causal [25]. Regardless of the incident waves, define a Hamiltonian $H$ :

$$
H=C \dot{z}^{2}+\lambda(\boldsymbol{\gamma} \cdot \boldsymbol{x}+\boldsymbol{\eta})
$$

$\lambda$ is a state vector with dimension $1 \times(n+2)$, which can be regarded as the Lagrange multipliers. $\boldsymbol{\gamma}$ and $\boldsymbol{\eta}$ have the same definitions in Eq. (4).

According to the Pontryagin's maximum principle, the optimal $\beta$ is the one maximizing the Hamiltonian at every time instant throughout $[0, T]$. The Hamiltonian is a linear function of $\beta$ so that $\beta$ 
must be the extremal values ( 0 or 1 ) to maximize the Hamiltonian. It is easy to find that the Hamiltonian reaches the maximum value on condition that

$$
\beta=\left\{\begin{array}{lc}
1 & \lambda_{2} c \dot{z}<0 \\
0 & \text { otherwise }
\end{array}\right.
$$

Assume that the random waves within the interval $[0, T]$ are already known, the time series of floater movement can be calculated. Subsequently, it is required to calculate $\lambda_{2}$ at each time instant and apply the latching control based on the binary sequence. Please note that the sate vector satisfies the following relationships.

$$
\begin{aligned}
& \dot{\lambda}_{i}=-\frac{\partial H}{\partial x_{i}}(t, \boldsymbol{x}, \beta), i=1,2, \ldots, n+2 \\
& \lambda(T)=\mathbf{0}
\end{aligned}
$$

Eq. (11) cannot be solved numerically like an initial value problem as the final condition is given here. In Ref [26], the canonical equations were solved based on the combination of discretization and dynamic programming. Zhong and Yeung [27] derived the control sequence with the so-called quadratic programming formulation. In our study, an iterative process is applied to calculate $\lambda$. Firstly, run the simulation with $\beta(t)=0$ to obtain the motions free of latching action by integrating Eq. (4) forward from $t=0$ to $t=T$. Subsequently, determine $\lambda$ by integrating Eq. (11) backwards from $t=T$ to $t=0(\lambda(T)=\mathbf{0}$ is now an initial condition). Based on Eq. (10), the control sequence $\beta(t)$ is derived.

Iterate the process with the updated control sequence until it converges.

\subsection{Real-time control}

The optimal command theory cannot be applied directly since it is impossible to know the wave forces over the entire interval. Nevertheless, one can forecast the short-term wave forces over a relatively short interval $[t, t+\Delta t]$ so that the optimal command theory can be used within the prediction interval. It is the basic idea of the real-time control strategy in this study.

Assuming that a wave force prediction model has been developed (the prediction model is the neural network in the present study), the procedure of the real-time control is illustrated in Fig. 3. At time instant $t_{i}$, collect the historical wave forces and perform the forecasting within $\left[t_{i+1}, t_{i+1}+\Delta t\right]$. Then, the control sequence $\beta(t), t \in\left[t_{i+1}, t_{i+1}+\Delta t\right]$ can be estimated with the optimal command theory. Please note that only the predicted control sequence $\beta\left(t_{i+1}\right)$ is adopted. At time instant $t_{i+1}$, apply the control action which has been predicted at the previous step and repeat the process again to predict the control action at time instant $t_{i+2}$. By repeating this algorithm step by step, the real-time control is implemented throughout the entire interval. Such a real-time control is also known as the receding horizon control or the model predictive control. Since the energy absorption is maximized over the predictive interval, the real-time control strategy is sub-optimal. 


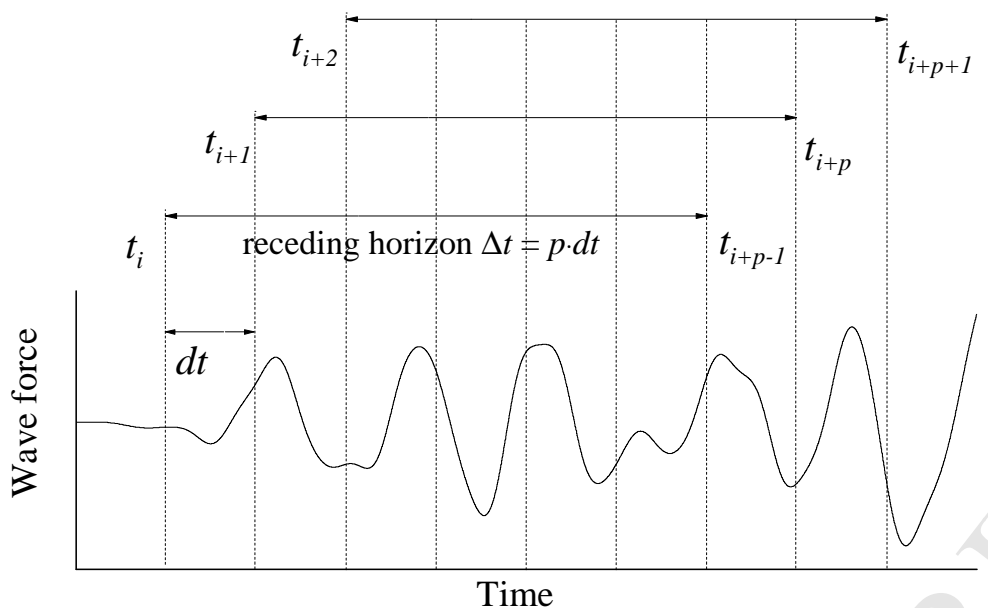

174

175

176

177

178

179

180

181

182

183

184

185

186

187

188

189

190

Fig. 3. Receding horizon control.

\section{Machine learning algorithm}

The machine learning algorithm is a member of the broad family of artificial intelligence. Its fundamental architecture is the artificial neural network, which is inspired by the biological neural network. The main idea of using machine learning to predict wave forces is that the neural network can learn and recognize the underlying relationships between the wave forces in the past and the coming wave forces through sufficient training examples.

\subsection{Neural network}

Fig. 4 illustrates the structure of an artificial neural network, which is composed of the input layer, the hidden layers, and the output layer. For the problem in this study, the input is namely the recorded wave forces in the past and the output is the prediction of wave forces over the predictive horizon. Several neurons are located in the layers to process the input signals. Two sets of parameters, weight $\boldsymbol{w}=\left(w_{11}, w_{12}, \ldots, w_{j i}, \ldots\right)$ and threshold $\boldsymbol{b}=\left(b_{11}, b_{12}, \ldots, b_{i j}, \ldots\right)$ are used to value the importance of the input signal. $w_{j i}$ and $b_{j i}$ are the parameters of the $i$-th neuron at the $j$-th layer. The estimated signal is subsequently normalized by the activation function before transferring to the next layer. The sigmoid function $\left(\sigma(x)=1 /\left(1+e^{-x}\right)\right)$ is selected here as the activation function.

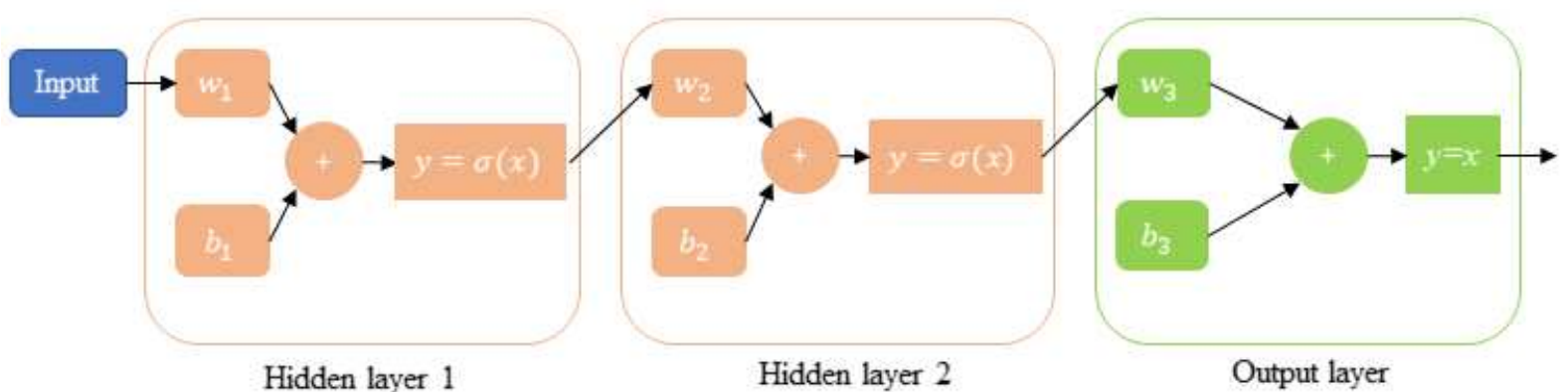

Fig. 4. Illustration of signal transformation between layers. 


\subsection{Training algorithm}

Before the neural network can be used for prediction, one has to tune the parameters $\boldsymbol{w}$ and $\boldsymbol{b}$ make it compatible with the problem concerned. This is fulfilled by training the network with a large number of examples (big data) so that it can learn how to predict the wave forces according to previous 'experiences'. A cost function $\Theta$ is introduced to estimate the performance of the neural network.

$$
\Theta(\boldsymbol{w}, \boldsymbol{b})=\frac{1}{2 L} \sum_{k=1}^{L}\left\|y^{k}-a^{k}\right\|^{2}
$$

$L$ is the number of training examples. $y^{k}$ and $a^{k}$ are the prediction output and the prediction target corresponding to the $k$-th training example. Given the examples, the objective of 'training' is to find the optimal parameters $\boldsymbol{w}$ and $\boldsymbol{b}$, which minimize the cost function $\Theta$. The optimal values are searched using the gradient descent method.

$$
\begin{gathered}
w_{j i}{ }^{\prime}=w_{j i}-\kappa \frac{\partial \Theta}{\partial w_{j i}} \\
b_{j i}{ }^{\prime}=b_{j i}-\kappa \frac{\partial \Theta}{\partial b_{j i}}
\end{gathered}
$$

$w_{j i}{ }^{\prime}$ and $b_{j i}{ }^{\prime}$ are the updated weight and threshold after training. $\kappa$ is the so-called learning rate, representing the sensitivity of the network to training example. In the case of $\kappa=0$, the network learns nothing from the training examples. Now, the key point is to acquire the gradient of cost function $\Theta$ at various layers. The backpropagation algorithm is used here to estimate the gradient of cost function. Start the backpropagation process by estimating the gradient at the output layer. Then the gradient at previous layers can be estimated. Continue the backpropagation algorithm until the gradients at all layer are obtained. Eventually, update parameters $\boldsymbol{w}$ and $\boldsymbol{b}$ with the acquired gradients.

We demonstrate the procedure of the training process with a simple case. Assume that the network has two hidden layers and each layer has only one neuron (see Fig. 4). The neural network is trained with a single example $(L=1)$. Denote $y_{0}$ the input to the network, the output of hidden layers is given by $y_{1}=\sigma\left(w_{1} y_{0}+b_{1}\right)$ and $y_{2}=\sigma\left(w_{2} y_{1}+b_{2}\right)$. The final output of the network is $y_{3}=w_{3} y_{2}+b_{3}$. Then, the deviation of cost function at the output layer is given by

$$
\delta_{3}=\frac{\partial \Theta}{\partial y_{3}} \cdot \sigma^{\prime}\left(w_{2} y_{1}+b_{2}\right)
$$

The deviation of cost function at the hidden layer is obtained with the backpropagation algorithm

$$
\delta_{i}=w_{i+1} \delta_{i+1} \sigma\left(w_{i} y_{i-1}+b_{i}\right), i=1,2
$$

At this point, the deviation of cost function at all layers are acquired. Afterward, the gradient of cost function at various layers is estimated with the following expressions 


$$
\begin{aligned}
& \frac{\partial \Theta}{\partial b_{i}}=\delta_{i} \\
& \frac{\partial \Theta}{\partial w_{i}}=y_{i-1} \delta_{i}
\end{aligned}
$$

223

226

230

With the gradient of cost function, the weights and thresholds are updated on the basis of Eq. (13). Iterate the above process until the pre-defined termination criteria are satisfied. Please refer to [28] for detailed procedures of the training process for more general cases.

\subsection{Estimation of prediction performance}

It is well-known the that successful implementation of real-time control requires accurate prediction of wave forces. Otherwise, the control performance may become bad [29]. The prediction ability of the trained neural network is checked through comparison with the traditional prediction methodology-grey model $\operatorname{GM}(1,1)$. The detailed procedure of using the grey model for prediction can be found in Appendix A. The random wave elevations measured in the Ref [30] is used to examine the prediction ability of the two models. The measured data were low-pass filtered to remove the high-frequency wave noise. The significant wave height of the random wave elevations is $0.04 \mathrm{~m}$ and the wave peak period is $1.13 \mathrm{~s}$. Although the neural network forecasts wave forces in the present research, it can be validated by the wave elevations since both variables are random signals by nature. We select $100 \mathrm{~s}$ of wave elevation measurement. The first $50 \mathrm{~s}$ data are used to train the neural network and the last $50 \mathrm{~s}$ are used for validation. Hong and Billings [31] proposed a simplified prediction index for quantitative assessment of the prediction

$$
\operatorname{Er}(\Delta t)=\frac{\int_{0}^{T} h^{2}(\tau) d \tau}{\int_{0}^{T} \bar{h}^{2}(\tau+\Delta t \mid \tau) d \tau}
$$

where $h(t)$ is the measured wave elevation at time instant $t ; \bar{h}(t+\Delta t \mid t)$ is the $\Delta t$ time ahead wave elevation predicted at time instant $t . E r<1$ indicates that the predicted values are larger than the target and vice versa. Er close to 1 represents a good prediction capacity.

Fig. 5 compares the prediction performances of the two models. The neural network behaves better than the grey model. The index $E r$ estimated by the neural network is generally around 1. Fig. 7 plots the predicted wave force histories. The force predicted by the neural network agrees well with the measurement whereas the grey model overestimates the wave force substantially. 


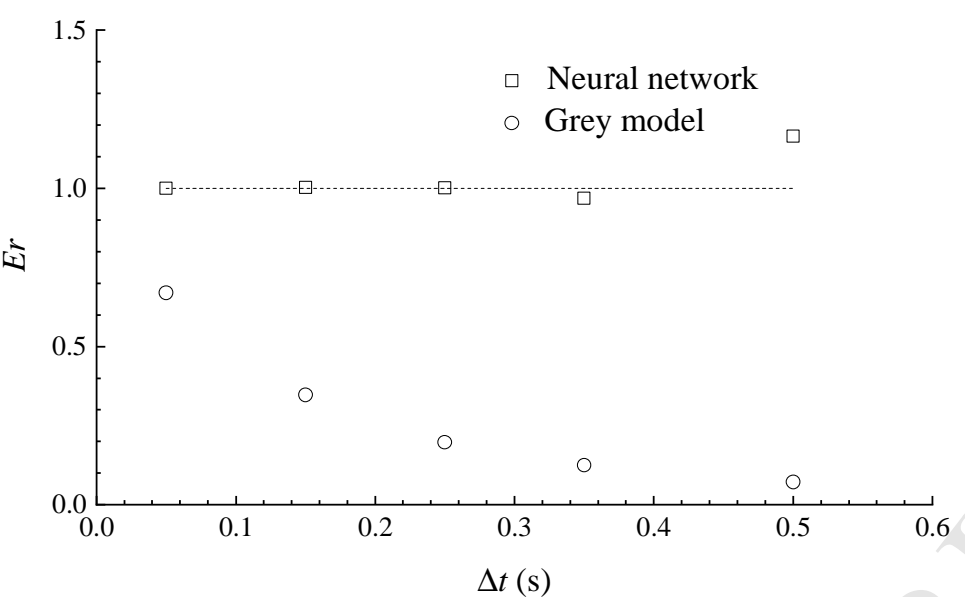

Fig. 5. Prediction index Er.

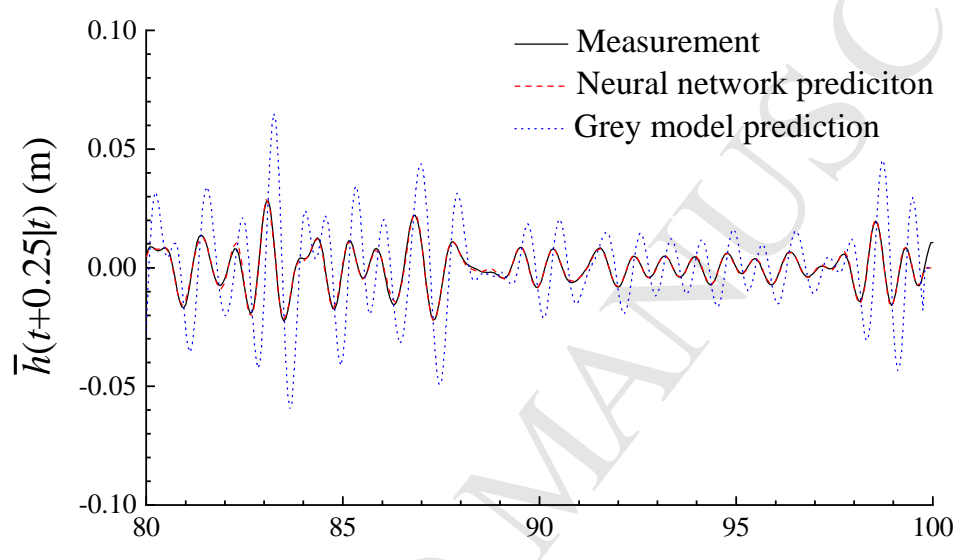

Time (s)

Fig. 6. Histories of predicted wave force with $\Delta t=0.25 \mathrm{~s}$.

\section{5. Validation}

252 Two aspects of validation are performed, namely the WEC dynamics and the control sequence 253 derivation.

\subsection{WEC dynamics}

255 Firstly, we validate the dynamic model in the absence of the latching control. The energy 256 absorption in a set of unit regular waves with various oscillation frequencies is simulated. The results 257 are compared with those estimated by frequency-domain hydrodynamic analysis programme Wadam

258 [32]. Please note that the point-absorber is a linear system without the latching control so that Wadam 259 is applicable here. The PTO system force is modeled with the 'additional damping' and 'additional 260 stiffness' options provided in Wadam. As displayed in Fig. 7, the agreement between the two simulation tools are very good. 


\subsection{Controller}

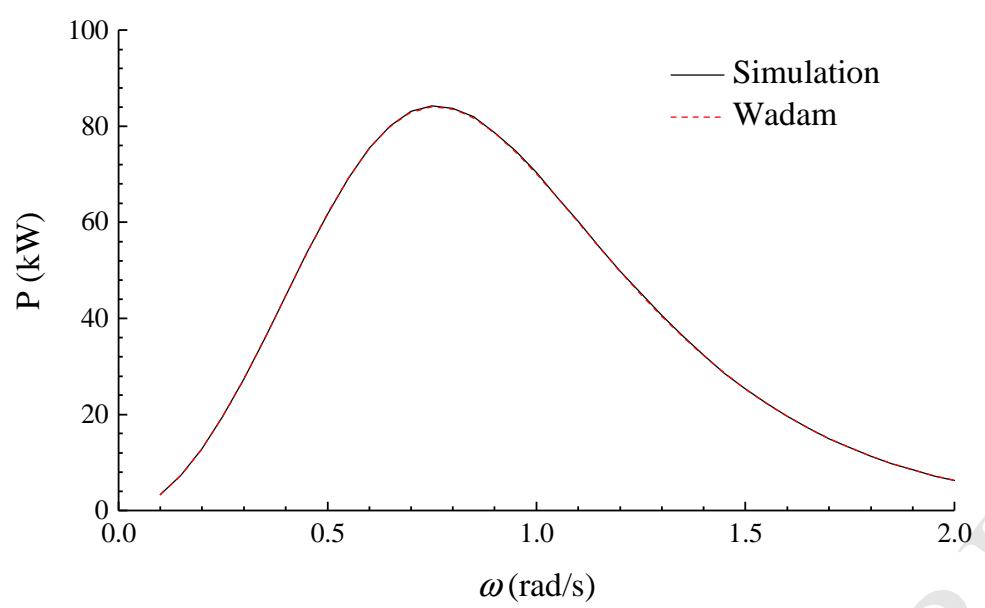

Fig. 7. Energy absorption in regular waves. $A=1 \mathrm{~m}$.

Babarit and Clement [13] presented a semi-analytical solution of the optimal latched duration in regular waves. According to their study, the optimal latched duration $t$ satisfies

$$
\begin{aligned}
y_{2} & =0 \\
\boldsymbol{Y} & =\left[y_{1}, y_{2}, \ldots, y_{n+1}, y_{n+2}\right] \\
& =-\left(\boldsymbol{I}+e^{\gamma \Delta}\right) \cdot\left(e^{\boldsymbol{\gamma}^{\prime} t}\right)^{-1} \times \operatorname{Re}\left\{\left[\left(\boldsymbol{I} e^{i \omega \Delta}-e^{\boldsymbol{\nu}}\right)(i \omega \boldsymbol{I}-\boldsymbol{\gamma})^{-1} \boldsymbol{\eta}\right] \times e^{i(\omega t+\varepsilon)}\right\} \\
\boldsymbol{\gamma}^{\prime} & =\left[\begin{array}{lll}
0 & 0 & 0 \\
0 & 0 & 0 \\
0 & 0 & \vec{A}
\end{array}\right], \Delta t=\pi / \omega-t
\end{aligned}
$$

268 where $\boldsymbol{I}$ is the identity matrix with dimension $(n+2) \times(n+2) . n$ is still the order of the system. The 269 detailed derivation of the semi-analytic solution can be found in Ref [13].

270 The latched duration suggested by Eq. (18) and simulated by the present controller are compared in Fig. 8. As shown, the optimal latched duration increases with the wave period, implying that a stronger latching action is needed in the case of long waves to regulate the response. Some discrepancies are observed because the absolute latching control (locking the WEC instantaneously) was used in Ref [13] whereas the present simulation applies a large damping force to lock the floater. 


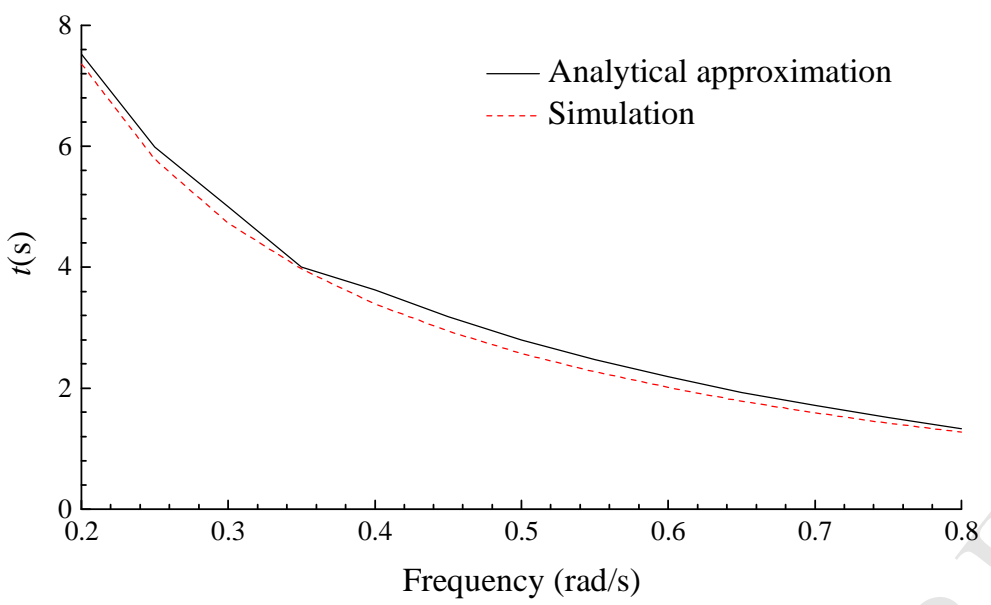

Fig. 8. Optimal latched durations in regular waves. $A=1 \mathrm{~m}$.

277

\section{Energy absorption}

The joint distribution model of stochastic waves proposed in Ref [33] is used to specify the random waves. The distribution model is based on the field measurement at Atlantic from 2001 to 2010. The marginal distribution of significant wave height $H_{s}$ follows a hybrid lognormal and Weibull distribution

$$
f_{H_{s}}(h)=\left\{\begin{array}{l}
\frac{1}{\sqrt{2 \pi} \sigma_{L H M} h} \cdot \exp \left[-\frac{1}{2}\left(\frac{\ln (h)-\mu_{L H M}}{\sigma_{L H M}}\right)^{2}\right] \quad h \leq h_{0} \\
\frac{\alpha_{H M}}{\beta_{H M}}\left(\frac{h}{\beta_{H M}}\right)^{\alpha_{H M}-1} \cdot \exp \left[\left(-\frac{h}{\beta_{H M}}\right)^{\alpha_{H M}}\right] \quad h>h_{0}
\end{array}\right.
$$

The conditional distribution of peak period $T_{p}$ at a given significant wave height follows a lognormal distribution. Detailed values of these parameters can be found in [33].

$$
f_{T_{p} \mid H_{s}}(t \mid h)=\frac{1}{\sqrt{2 \pi} \sigma_{L T C} t} \cdot \exp \left[-\frac{1}{2}\left(\frac{\ln (t)-\mu_{L T C}}{\sigma_{L T C}}\right)^{2}\right]
$$

$$
\mu_{L T C}=c_{1}+c_{2} \cdot h^{c_{3}}
$$

$$
\sigma_{L T C}^{2}=d_{1}+d_{2} \cdot \exp \left(d_{3} \cdot h\right)
$$

A set of significant wave heights are selected at first, and the most probable peak periods are subsequently determined based on the joint model. The selected random wave conditions are listed in Table 1. $4000 \mathrm{~s}$ of random wave elevations are generated in each simulation case and the response in the first $400 \mathrm{~s}$ is cut off to eliminate the transient effect arising in early simulation stage.

Table 1 Wave conditions

\begin{tabular}{cccc}
\hline & Case 1 & Case 2 & Case 3 \\
\hline$H_{s}(\mathrm{~m})$ & 2 & 4 & 6 \\
$T_{p}(\mathrm{~s})$ & 11.11 & 12.33 & 13.27 \\
\hline
\end{tabular}




\subsection{Regular waves}

We first investigate the energy absorption in regular waves. Since the waves are regular, the wave forces over the predictive horizon are given rather than predicted here. The predictive horizon is set to $0.2 \cdot(2 \pi / \omega)$. The wave amplitude $A$ is $1 \mathrm{~m}$. Fig. 9 plots the sensitivity of energy absorption to wave oscillation frequency. The control effect on the energy absorption is noticeable, especially within the low-frequency range.

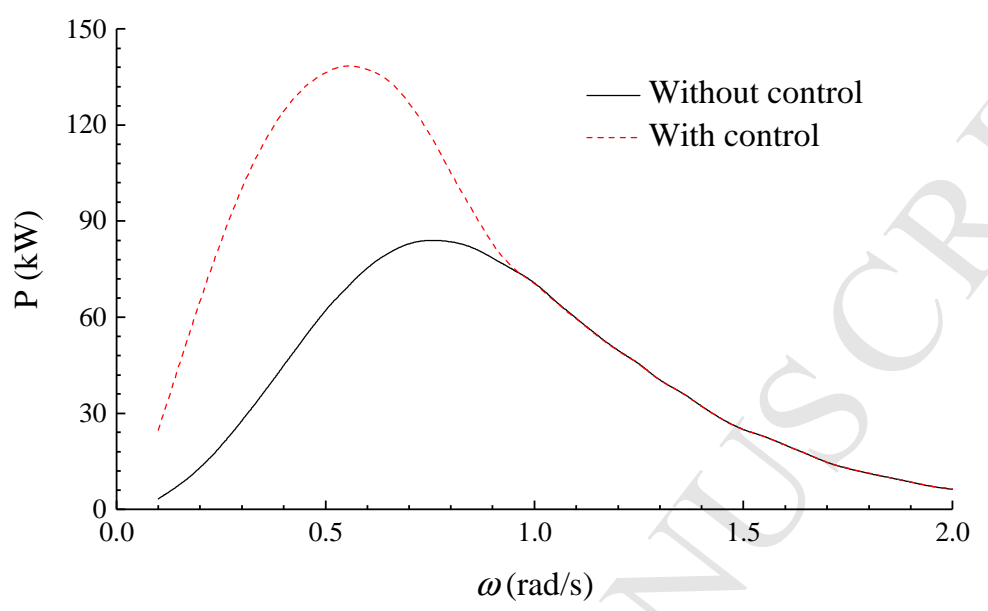

Fig. 9. Average energy absorption in regular waves. $A=1 \mathrm{~m}$.

Fig. 10 displays the phase portrait of the responses. It is obvious that the velocity phase is regulated by the controller. The latching control is a kind of phase control by nature and it maximizes energy absorption by tuning velocity phase. Fig. 11 displays the controlled motion and its relative phase with respect to the wave forces. According to the velocity time-series, the point-absorber is latched and released alternately so that the response is a succession of locked and ramp stages. Due to the regulation of the controller, the velocity and the wave forces are in phase so that the wave forces will always accelerate the floater and the floater carries more kinetic energy as a result. This property has been widely used as the criterion to validate latching control since the work of Budal and Falnes [12]. Therefore, Fig. 11 can be used to validate the present numerical model as well.

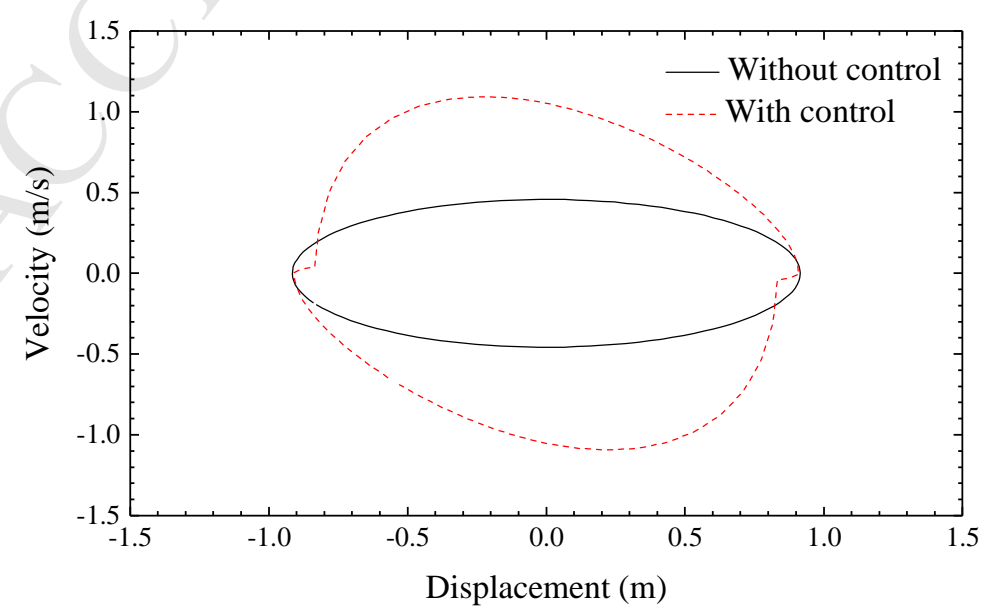




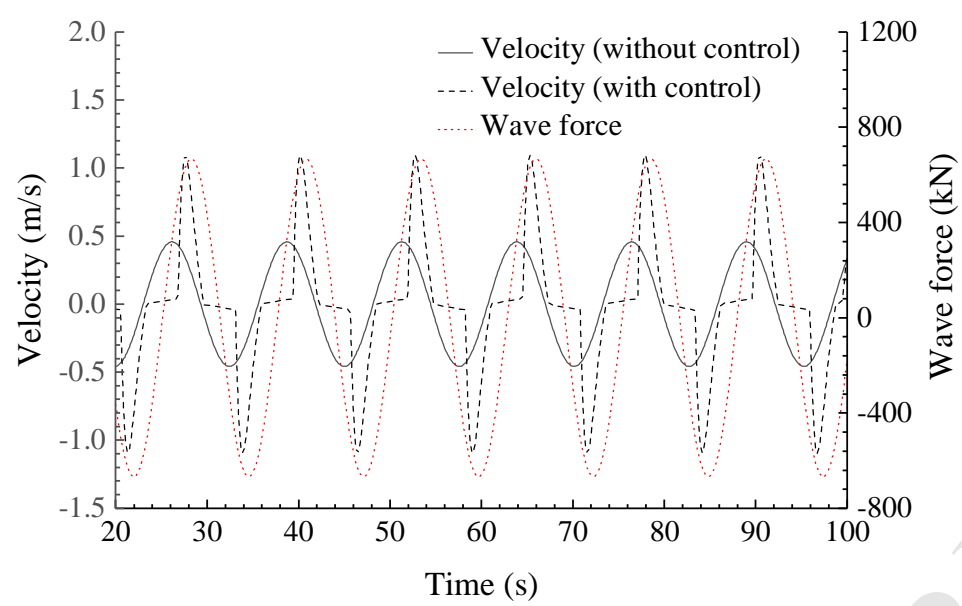

Fig. 11. Responses of the floater in regular wave. $A=1 \mathrm{~m}, \omega=0.5 \mathrm{rad} / \mathrm{s}$.

Nevertheless, it seems that the controller is not effective at all within the high-frequency range. According to Fig. 8, the optimal latched duration reduces gradually when the wave frequency increases and reduces to nearly zero in the case of very high-frequency waves. It implies that it is unnecessary to regulate the point-absorber in high-frequency wave and thereby the energy absorption is hardly increased. As pointed out in Ref [34], the optimal duration of a single locked stage is close to half of the natural period of the WEC on condition that the PTO damping coefficient $C$ is sufficiently small (weak damping). Therefore, the solution of the control sequence is only available when the wave period is sufficiently long. Although the damping coefficient $C$ is very large in our model and the sub-optimal receding horizon control is used here, this property can still help to interpret why the latching control is merely effective in low-frequency waves.

\subsection{Irregular waves}

In the real oceans, the waves are random and oscillate with multiple frequencies so that the wave forces are unknown. To examine the validity of the smart controller, we run simulations in regular waves where the short-term future wave forces are predicted by the neural network. Fig. 12 plots the histories of floater velocity and wave forces. Like regular wave case, the point-absorber is also locked frequently in random waves. Whenever the wave force and the velocity are reverse, the floater is locked to avoid the slowing down of velocity. Once the floater is released, the velocity builds up rapidly in a short time. Owing to the regulation of the controller, the velocity is generally in phase with the wave forces. 


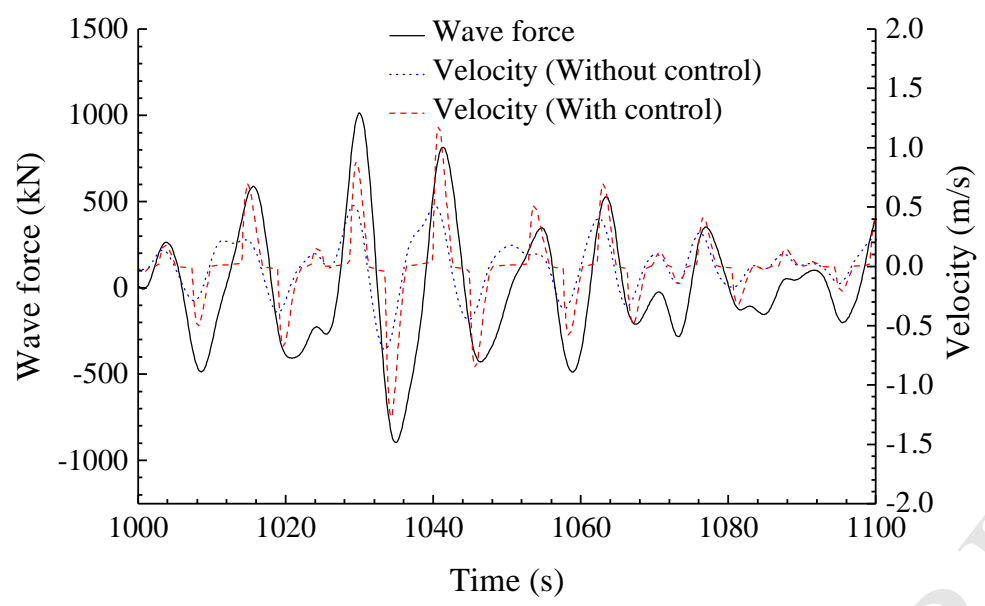

Fig. 12. Responses of the point-absorber, Case1.

The control effect on the energy absorption is illustrated in Fig. 13. Although the point-absorber is locked frequently, and the PTO systems stop working during the locked duration, the energy extraction ramps once the point-absorber is set free since the velocity runs up rapidly during the ramp stage. It leads to the enhancement of the average energy absorption. The 1-hr average energy harvesting under various wave conditions is presented in Fig. 14. Generally, the point-absorber produces $60 \%$ 80\% more electrical energy with the smart controller. It manifests that the neural network can be successfully applied in the real-time control of WEC.

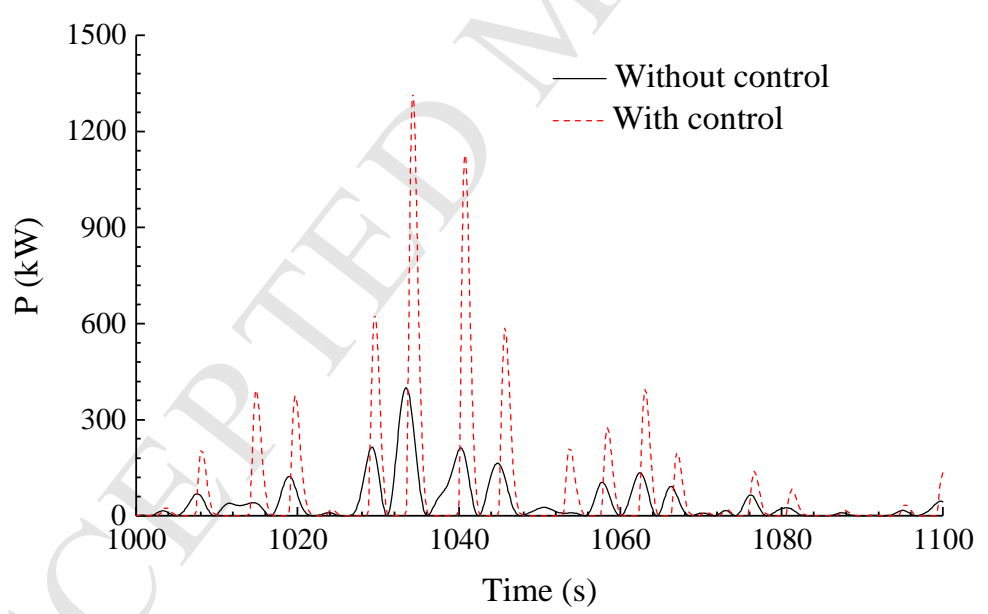

Fig. 13. Power capture, Case1. 


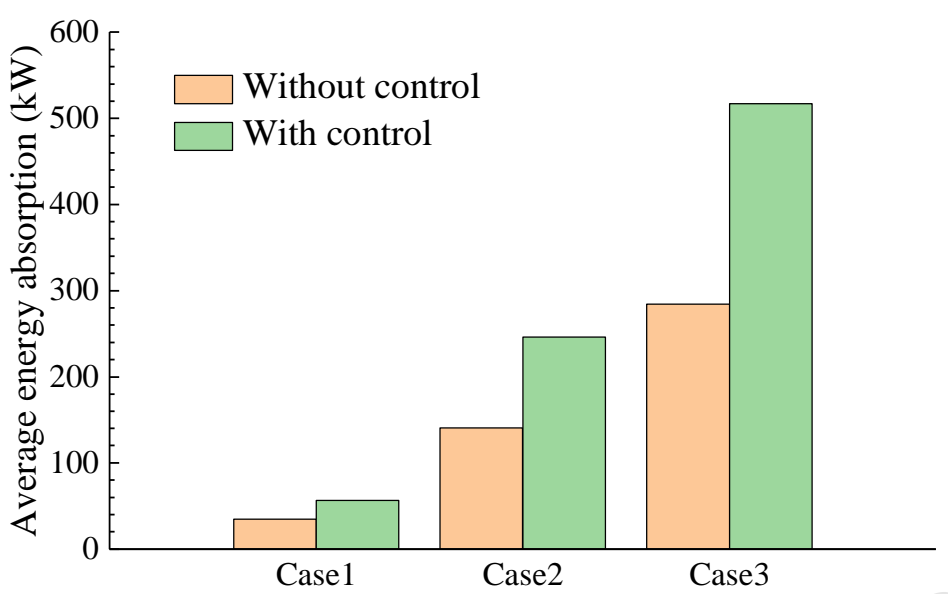

Fig. 14. Average energy harvesting.

As discussed in Section 3.2, the model predictive control can be regarded as a kind of sub-optimal control. Consequently, the efficiency of the controller is evaluated by comparing with the optimal one. Please note that by implementing the optimal control it inherently implies that the wave forces over the entire interval are already known. Fig. 15 displays the control sequence using the two control schemes. The control sequence predicted by the neural network is close to the optimal one, indicating that the prediction accuracy of the neural network is satisfactory. Some discrepancies are observed since the prediction deviation is unavoidable. Also, the model predictive control is sub-optimal by nature and it cannot acquire the optimal sequence even in the absence of prediction deviation.

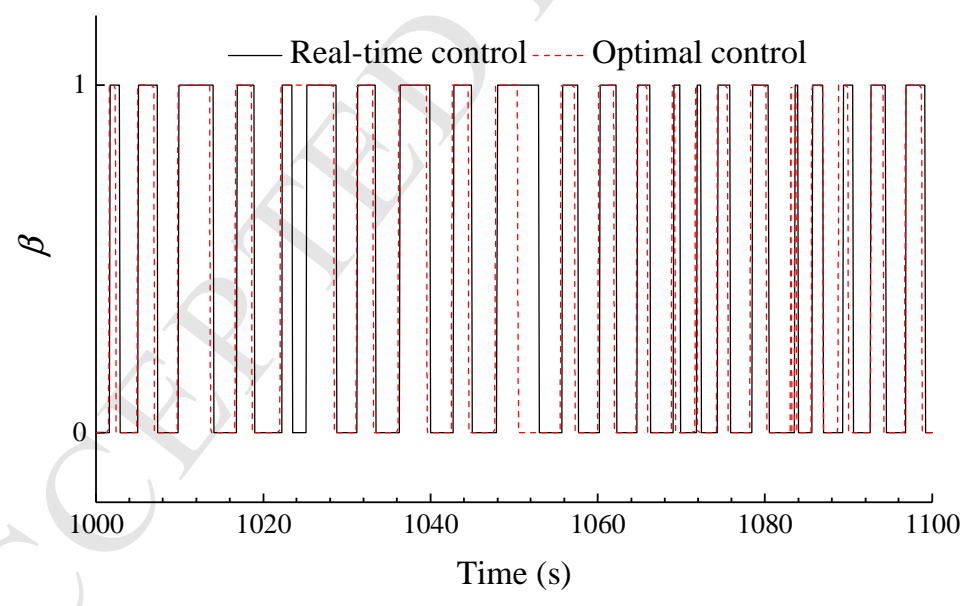

Fig. 15. Control sequence, Case 1.

Table 2 lists the average energy absorption obtained using the model predictive control and the optimal control. Generally, the model predictive control underestimates the energy harvesting by no more than $9 \%$. Although the real-time control is sub-optimal, the control efficiency is still satisfactory even if the wave forces are predicted.

Table 2 Average energy absorption estimated by real-time control and optimal control.

\begin{tabular}{ccc} 
& Real-time control & Optimal control \\
\hline Case1 & $56 \mathrm{~kW}$ & $61 \mathrm{~kW}$ \\
Case2 & $246 \mathrm{~kW}$ & $266 \mathrm{~kW}$ \\
Case3 & $517 \mathrm{~kW}$ & $571 \mathrm{~kW}$ \\
\hline
\end{tabular}




\subsection{Energy absorption with different prediction models}

To demonstrate the advantage of the neural network against traditional prediction approach, the neural network is compared with the grey model $\operatorname{GM}(1,1)$. The control efficiency will be evaluated to show the accuracy of the two prediction models.

Table 3 lists the average power extraction of the point-absorber using different wave force prediction models. As shown, the control efficiency is reduced substantially if the wave forces are predicted by the grey model. Since the point-absorber is subject to identical wave forces, the discrepancies on the control performance are completely caused by the prediction error. It thus implies that the neural network is a more reliable prediction approach.

$370 \quad$ Table 3 Average power extraction with different prediction models

\begin{tabular}{cccc}
\hline & Case1 & Case2 & Case3 \\
\hline Neural network & $56 \mathrm{~kW}$ & $246 \mathrm{~kW}$ & $517 \mathrm{~kW}$ \\
GM $(1,1)$ & $50 \mathrm{~kW}$ & $201 \mathrm{~kW}$ & $429 \mathrm{~kW}$ \\
\hline
\end{tabular}

Fig. 16 plots the optimal control sequence and the predicted sequence with respect to the two prediction models. Due to the unavoidable prediction deviation, the predicted control sequence is somewhat different from the optimal one. Nevertheless, it is easy to find that the sequence predicted by the neural network is closer to the optimal one, attributing to the better prediction capacity. A more appropriate control sequence indicates the point-absorber will be released and locked at the right time instants and thereby the point-absorber extracts more power with the neural network 

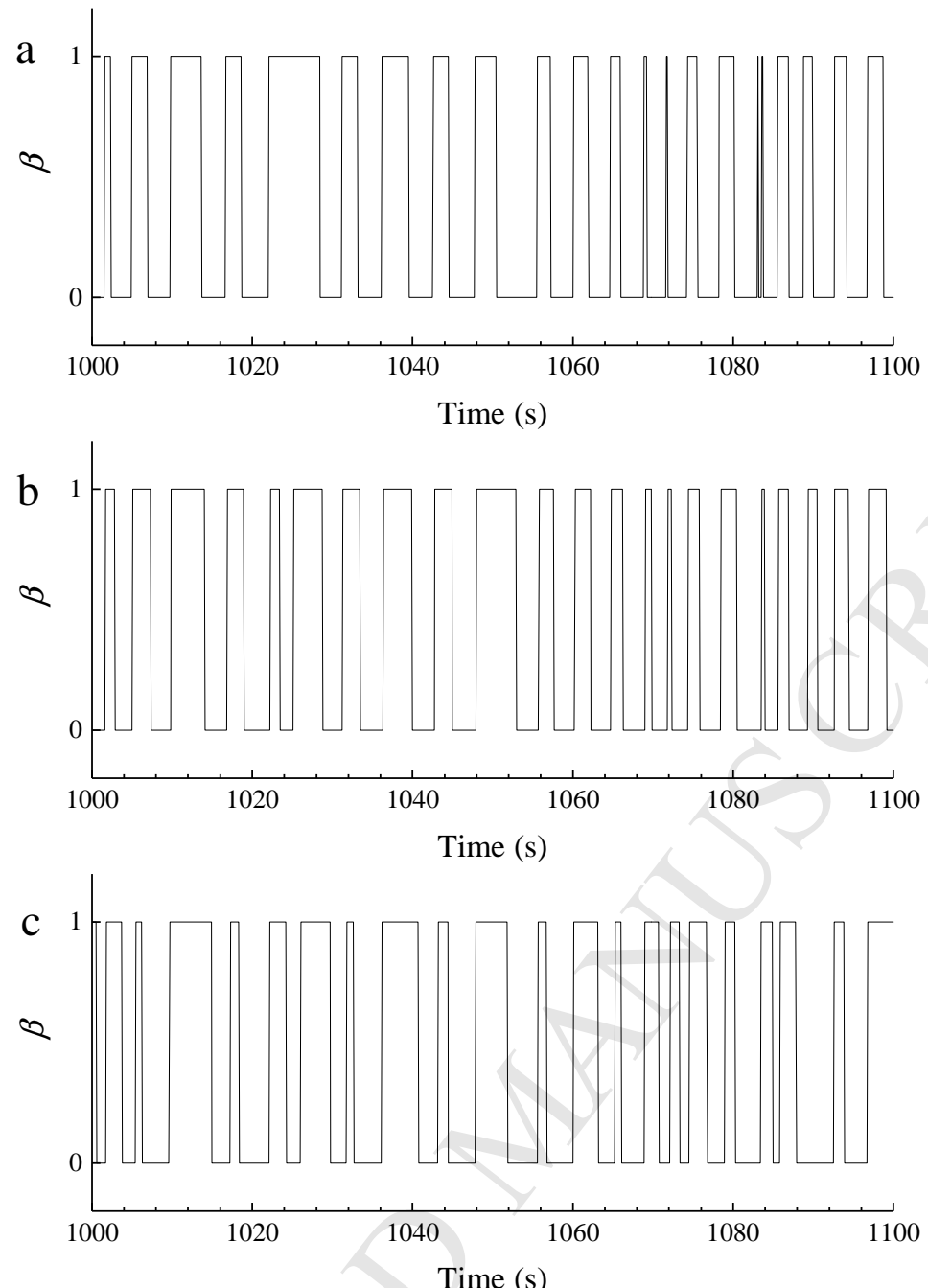

Time (s)

Fig. 16. Control sequence forecasted by different prediction models, Case1. (a) Optimal; (b) Neural network; (c) GM(1,1).

\section{Conclusions}

Wave force prediction is necessary for the implementation of WEC real-time control. As proved in previous studies, the prediction deviation will reduce the efficiency of real-time control and thereby an accurate wave force prediction method is essential. In our work, an artificial neural network is developed to predict the short-term wave forces. Based on the developed neural network, the real-time latching control is implemented to a heaving point-absorber to maximize the energy extraction in random waves.

The neural network is trained using the machine learning algorithm. The training process is based on the backpropagation algorithm, in which the gradient of cost function at various layers are estimated to update the weights and the thresholds. Based on a large number of training examples, the weights and thresholds identifying the neural network are optimized gradually until the outputs agree well the desired targets.

The point-absorber harvests more power with the real-time control, which is attributed to the tuning of velocity phase. The controller tunes the velocity by locking and releasing the floater 
alternately so that the velocity and the wave forces are in phase. In that case, the wave forces always accelerate the point-absorber and the floater carries more kinetic energy. For the random wave conditions considered in this study, the energy absorption is increased by $60 \% \sim 80 \%$ with the smart controller. Owing to the good prediction capacity of the neural network, the control efficiency is satisfactory, just slightly lower than the optimal level.

\section{Acknowledgment}

This work is supported by China Scholarship Council [Grant No. 201506230127]. The authors are grateful for their financial support.

\section{References}

[1] Bahaj AS, Batten WMJ, McCann G. Experimental verifications of numerical predictions for the hydrodynamic performance of horizontal axis marine current turbines. Renew Energ. 2007;32(15):2479-90.

[2] Li L, Gao Y, Hu Z, Yuan Z, Day S, Li H. Model test research of a semisubmersible floating wind turbine with an improved deficient thrust force correction approach. Renew Energ. 2018;119:95-105. [3] Muliawan MJ, Karimirad M, Moan T. Dynamic response and power performance of a combined Spar-type floating wind turbine and coaxial floating wave energy converter. Renew Energ. 2013;50:47-57.

[4] Li L, Gao Y, Yuan ZM, Day S, Hu ZQ. Dynamic response and power production of a floating integrated wind, wave and tidal energy system. Renew Energ. 2018;116:412-22.

[5] He F, Huang ZH, Law AWK. An experimental study of a floating breakwater with asymmetric pneumatic chambers for wave energy extraction. Appl Energ. 2013;106:222-31.

[6] Falcao AFO, Henriques JCC. Oscillating-water-column wave energy converters and air turbines: A review. Renew Energ. 2016;85:1391-424.

[7] Stansby P, Moreno EC, Stallard T. Large capacity multi-float configurations for the wave energy converter M4 using a time-domain linear diffraction model. Appl Ocean Res. 2017;68:53-64.

[8] Zhang XT, Yang JM. Power capture performance of an oscillating-body WEC with nonlinear snap through PTO systems in irregular waves. Appl Ocean Res. 2015;52:261-73.

[9] Xiao XL, Xiao LF, Peng T. Comparative study on power capture performance of oscillating-body wave energy converters with three novel power take-off systems. Renew Energ. 2017;103:94-105.

[10] Babarit A, Guglielmi M, Clement AH. Declutching control of a wave energy converter. Ocean Eng. 2009;36(12-13):1015-24.

[11] Tom NM, Yu YH, Wright AD, Lawson MJ. Pseudo-spectral control of a novel oscillating surge wave energy converter in regular waves for power optimization including load reduction. Ocean Eng. 2017;137:352-66. 
[12] Budal K, Falnes J. Interacting point absorbers with controlled motion, in Power from Sea Waves: BM Count, Academic Press, 1980.

[13] Babarit A, Clement AH. Optimal latching control of a wave energy device in regular and irregular waves. Appl Ocean Res. 2006;28(2):77-91.

[14] Henriques JCC, Gato LMC, Falcao AFO, Robles E, Fay FX. Latching control of a floating oscillating-water-column wave energy converter. Renew Energ. 2016;90:229-41.

[15] Mcculloch WS, Pitts W. A Logical Calculus of the Ideas Immanent in Nervous Activity. B Math Biol. 1943;5(4):115-33.

[16] Hinton GE, Osindero S, Teh YW. A fast learning algorithm for deep belief nets. Neural Comput. 2006;18(7):1527-54.

[17] Lv YS, Duan YJ, Kang WW, Li ZX, Wang FY. Traffic Flow Prediction With Big Data: A Deep Learning Approach. Ieee T Intell Transp. 2015;16(2):865-73.

[18] Islam MP, Morimoto T. Non-linear autoregressive neural network approach for inside air temperature prediction of a pillar cooler. Int J Green Energy. 2017;14(2):141-9.

[19] Pourzangbar A, Losada MA, Saber A, Ahari LR, Larroude P, Vaezi M, et al. Prediction of nonbreaking wave induced scour depth at the trunk section of breakwaters using Genetic Programming and Artificial Neural Networks. Coast Eng. 2017;121:107-18.

[20] Ebtehaj I, Bonakdari H, Moradi F, Gharabaghi B, Khozani ZS. An integrated framework of Extreme Learning Machines for predicting scour at pile groups in clear water condition. Coast Eng. 2018;135:1-15.

[21] Vicente PC, Falcao AFO, Justino PAP. Nonlinear dynamics of a tightly moored point-absorber wave energy converter. Ocean Eng. 2013;59:20-36.

[22] Cummins W. The impulse response function and ship motions. Washington DC: David Taylor Model Basin; 1962. p. 101-9.

[23] Faltinsen OM. Sea Loads on Ships and Offshore Structures: Cambridge University Press, 1993. [24] Li L, Hu ZQ, Wang J, Ma Y. Development and Validation of an Aero-hydro Simulation Code for Offshore Floating Wind Turbine. J Ocean Wind Energy. 2015;2(1):1-11.

[25] Falnes J. Ocean waves and oscillating systems: linear interactions including wave-energy extraction: Cambridge university press, 2002.

[26] Li G, Weiss G, Mueller M, Townley S, Belmont MR. Wave energy converter control by wave prediction and dynamic programming. Renew Energ. 2012;48:392-403.

[27] Zhong Q, Yeung RW. An Efficient Convex Formulation for Model-Predictive Control on WaveEnergy Converters. Conference An Efficient Convex Formulation for Model-Predictive Control on Wave-Energy Converters. American Society of Mechanical Engineers, p. V010T09A35-VT09A35. [28] Nielsen M. Neural Networks and Deep Learning2017.

[29] Li L, Yuan Z, Gao Y, Zhang X. Wave force prediction effect on the energy absorption of a wave energy converter with real-time control. IEEE T Sustain Energ. 2018 (In Press). 
465 [30] Li L, Gao Y, Hu ZQ, Yuan ZM, Day S, Li HR. Model test research of a semisubmersible floating

466 wind turbine with an improved deficient thrust force correction approach. Renew Energ. 2018;119:95-

467105.

468 [31] Hong X, Billings S. Time series multistep-ahead predictability estimation and ranking. Journal of

469 Forecasting. 1999;18(2):139-49.

470 [32] Veritas DN. WADAM-Wave Analysis by Diffraction and Morison Theory. SESAM user's

471 manual, Høvik1994.

472 [33] Li L, Gao Z, Moan T. Joint Distribution of Environmental Condition at Five European Offshore

473 Sites for Design of Combined Wind and Wave Energy Devices. J Offshore Mech Arct. $474 \quad 2015 ; 137(3): 031901$.

475 [34] Babarit A, Duclos G, Clement AH. Comparison of latching control strategies for a heaving wave 476 energy device in random sea. Appl Ocean Res. 2004;26(5):227-38. 


\section{Appendix A}

481

Step1: At time instant $t_{i}$, collect the raw data $\boldsymbol{X}$ over the past few seconds

482

$$
\boldsymbol{X}=\left(x_{1}, x_{2}, \ldots, x_{n}\right)
$$

483 Step2: Generate an accumulated series $\boldsymbol{Y}$

484

$$
\boldsymbol{Y}=\left(y_{1}, y_{2}, \ldots, y_{n}\right)
$$

$$
y_{k}=\sum_{i=1}^{k} x_{i}, k=1,2, \ldots, n
$$

Step3: Generate the so-called background series $\boldsymbol{Z}$

486

$$
\begin{aligned}
& \boldsymbol{Z}=\left(z_{2}, z_{3}, \ldots, z_{n}\right) \\
& z_{k}=\left(y_{k}+y_{k-1}\right) / 2
\end{aligned}
$$

487 Step4: Set the grey differential formula

$$
x_{k}+a z_{k}=b, k=2,3, . ., n
$$

489 Step5: Estimate parameters $a$ and $b$ with the least square method

490

$$
\left[\begin{array}{l}
a \\
b
\end{array}\right]=\left(\boldsymbol{A}^{T} \boldsymbol{A}\right)^{-1} \boldsymbol{A}^{T} \boldsymbol{B}
$$

$$
\boldsymbol{A}=\left[\begin{array}{cc}
-z_{2} & 1 \\
-z_{3} & 1 \\
\vdots & \vdots \\
-z_{n} & 1
\end{array}\right], \boldsymbol{B}=\left[\begin{array}{c}
x_{2} \\
x_{3} \\
\vdots \\
x_{n}
\end{array}\right]
$$

491

Step6: Establish the first order-one variable grey model $\operatorname{GM}(1,1)$ to predict the random signal 492 within interval $\left[t_{i+1}, t_{i+p}\right]$

493

$$
\begin{aligned}
& \hat{x}_{n+p}=\hat{y}_{n+p}-\hat{y}_{n+p-1} \\
& \hat{y}_{n+p}=\left(y_{1}-\frac{b}{a}\right) e^{-a(n+p-1)}+\frac{b}{a}
\end{aligned}
$$

494 where $\hat{x}_{n+p}$ is the predicted data at time instant $t_{i+p}$. 
A smart real-time controller is developed.

The smart controller uses artificial neural network to predict short-term wave forces.

The neural network is trained by the deep learning algorithm.

The prediction accuracy of the neural network is satisfactory.

The smart controller enhances the energy absorption substantially. 